\title{
Mandibular etymologies
}

\author{
L. Cascarini ${ }^{1}$
}

\begin{abstract}
Many of us can remember how much our vocabulary grew as undergraduates and indeed has continued to grow as dental professionals. For many of us not trained in Latin and Greek we have often been too busy learning what the word means to understand why it means what it does. This article aims to clarify things a little. It explains where words relating to the mandible come from, why we use them now and also shows how they are related to other similar words in everyday use.
\end{abstract}

Mandible comes from the Latin mandere - to chew with; -bula by means of. Early anatomists used the word maxilla for both jaws as L maxilla means just jaw bone and is not at all specific to upper or lower, so they would have referred to the superior and inferior maxilla. Later anatomists decided to reserve maxilla for the upper jaw and used mandible for that which chews against it. In the UK we still use the term intermaxillary fixation or IMF, though in the US maxillomandibular fixation or MMF would be considered correct. The Greek for jaw is gnathos hence the terms orthognathic, L ortho- straight; prognathic L pro- forward; and retrognathic L retro- backward. The gnathion is the inferior border of the mandible in the midline. Gnathos is not thought to be the origin of gnash as in gnashing of teeth, which is probably from Old Norse gnastan: to gnash. Likewise gnaw is also from old Norse gnagna: to chew or nibble.

The condyle and epicondyle are from

\footnotetext{
'Dept of Oral and Maxillofacial Surgery, Guy's Hospital, St Thomas' Street, London, SE1 9RT Correspondence to: Luke Cascarini

Email: Icascarini@hotmail.com
}

\section{Refereed Paper}

Accepted 27 February 2007

DOI: $10.1038 /$ bdj.2007.733

${ }^{\oplus}$ British Dental Journal 2007; 203: 209-210 the Greek kondylos - a knuckle or knob - used by anatomists for rounded articular surfaces such as in the lower femur, the elbow and of course the temporomandibular joint. Epicondyle is kondylos with epi- Greek for in addition to. The origin of coronoid is less clear. The Latin word corona means a garland, wreath or crown and gives the name to the coronary arteries which encircle the heart like a crown and HM Coroner, originally the King's man or the man of the Crown, but this is not thought to be the origin. Nor is the Latin word coris meaning heart, as in cor pumonale. The most likely explanation for the origin of this beak-like prominence is the Greek word korone - a sea crow or anything curved like the beak of a crow.

The pterygoid fovea (or fossa) is named from the Greek pterix - a wing and oeidos - having the form of, as in pterodactyl. Not because the fossa is like a wing but because the lateral pterygoid muscles are attached here and they are named after their other insertion, the wing-like pterygoid plates of the sphenoid bone (Gk wedge like). Fovea is Latin for a small pit (fossa is Latin for ditch).

The sigmoid notch between the coronoid and condylar processes is named from the Greek sigma meaning the letter S, which in its lower case is the symbol, similar to the modern English C. The sigmoid notch is clearly more C-shaped than S-shaped. It is unclear whether the term was given to the sigmoid colon because it was considered S-shaped or C-shaped anatomically one could make a good case for either. There are other similar uses of letters in anatomy such as: hyoid, the U-shaped bone in the neck; lambdoid the Gk L-shaped or - shaped skull suture; and deltoid muscle which is Gk D-shaped or ).

The mandibular ramus is from $L$ ramus - a branch or bough, used for structures emanating like a branch from a larger structure. Some purists like to call the marginal mandibular branch of the facial nerve the 'ramus mandibularis'.

Gonion is the lower outermost part of the angle of the mandible and is from the Greek gonia meaning angle. Hence orthopaedic surgeons use goniometers to measure joint angles.

Alveolar bone has an uncertain etymology. The Latin word alveolus means a small tray or basin and was also applied to a game in which pebbles were held in small depressions. By extension, alveolus came to mean a small cavity or compartment. Vesalius (1514-1564) was probably the first person to apply the term to a tooth socket, hence alveolar bone for that which supports teeth. Only later, in the nineteenth century, 
did alveolus become used for the air sacs of the lung. Another possible derivation is that alveolus is the diminutive of $L$ alveus - a cavity, related to L alvus - belly and Gk aulos - a hollow tube.

The lingula is the diminutive form of L lingua - a tongue and refers to the little tongue of bone at the mandibular foramen. Lingua is also the origin of words like language and expressions like speaking in tongues. The Greek for tongue is glossa as in glossopharyngeal and also gives us glossary, glossitis, glottis and polyglot.

The mylohyoid line or groove is a combination form. Mylos is Greek for a grinding wheel, a millstone or the grinding back teeth, the molars. Hyoid is given above.

The mental foramen from $\mathrm{L}$ mentum - the chin and not in any way related to the mind, which comes from
L mens, mentis - the mind or intellect. Foramen is from L an opening or orifice, L forare to bore. Genial tubercles are from $L$ gena which derives from the Greek genus - the jaw. Tubercle means a pimple or small swelling, diminutive of tuber - a hump.

The digastric fossa is from $\mathrm{L}$ gaster, belly, and di- two hence its use for the muscle with two bellies, anterior and posterior, with a tendon between. 